\title{
INDEPENDENT OCULAR POLYNEURITIS AFTER DANDY FEVER VIRAL ENTEROBIASIS
}

\author{
Travis Evans \\ Remington College Campus Columbia
}

\begin{abstract}
:
We report a case of optic rub or when infectious disease infection, a development terribly seldom seen. We report a fifty four years recent male patient, conferred with headache, giddiness and cut vision in left eye when having had a benign type of break bone fever two weeks before. The rape tic intervention with steroid hormone medical care resulted in benign evolution. The optic rub or is incredibly rare in infectious disease viral infection.
\end{abstract}

\section{INTRODUCTION}

Dandy fever may be an infection transmitted by the feminine two-winged insects A.aegyptiand A.albopictushas diurnal habits. The dandy fever virus is that the Flaviridafamily, arbor virus group B and known four serotypes (1, 2, 3 and 4). Dandy fever will surface in many ways from a straightforward cold to a virus infection and shock, threatening life. The clinical features of viral dandy fever infection can be classified into five presentations: non-specific febrile ill ness, dandy fever, virus infection dandy fever, virus infection dandy fever with shock syndrome, and different uncommon syndromes like brain disease and hepatitis

(1). There are few reports of the neurological manifestations of dandy fever within the sort of severe rub or. The medicine involvement in sickness\} infection is seen once the acute disease has given associate degreed is believed to be an immune - mediate.

(2).Expression the reared some ocular manifestations in dandy fever.

(3). including the most common are: retro-orbitalpainretinal

(4) Hemorrhages, subconjunctival hemorrhage manifestations such (5), (6), (7), as optic inflammation endovascular maculopathy choroid effusion

(8), Roth spots redness, exudative detached retina and anterior

(9) We have a tendency to describe a case that showed low visual modality, papillitis and field of regard alterations once infection thanks to dandy fever. 


\section{The American Journal of Applied}

\section{sciences}

\section{Case history:}

A 54-year-old man conferred with history of giddiness, headache and reduce vision in left eye since 2 days. Fortnight before, the patient was admitted within the same hospital for infectious disease that was proved by presence of I Manti bodies. He was treated guardedly with blood vessel fluids, electrolytes, and aldohexose. His recovery was uneventful. Two weeks after discharge, patient old gradual loss of vision in his left eye, giddiness and headache. On Physical examination, he was afebrile with vital organ were traditional. On general examination apart from abnormally there were no abnormal findings. Extra ocular motility was normal without pain. No other neurological deficits found. On ocular examination, he had visual sense of investigating fingers near to face with correct projection of rays in left eye and 6/6 in right eye. Aperture examination disclosed relative sensory aperture defect (RAPD) on the proper aspect. Remainder of the anterior phase examination was routine in each eyes. Bodily structure examination of the left eye showed Associate in Nursing elevated point with hyperemia, obliterate cup, blood vessel engorgement and per papillary edema. The right eye bodily structure was WNL.

\section{Discussion:}

In dandy fever infection the onset is sharp with a high fever, headache and a great deal of pain within the body. A sense of intense weariness, lack of appetency, and sometimes nausea and inborn reflex are common. Erie theme might occur with blemishes, kind of like those of contagious disease or measles, and itch within the body. Some trauma might occur (usually within the nose or gums).

The designation of dandy fever is clinical. Medical science ought to be done in the main to regulate medical specialty police work, since it's typically useless within the conduct of treatment. Antibodies of the immune globulin kind against dandy fever virus are detectable from the sixth day and last up to ninety days, since the IgM is detected within the 1st days of 
the malady till 2 or 3 days when the infection. The main ocular changes reported within the literature associated with dandy fever embody retinal trauma and macula, periodical hemorrhage, Roth spots, diffuse retinal demi, vitreous cells, optical disc margin blurring, bodily fluid visual defect, choroid effusion and non-(1).specific maculopathy Optic rub or is AN inflammatory, infectious, or demyelinating method of the cranial nerve that will manifest as retro bulbar polyneuritis, papillitis, or neuroretinitis. Papillitisis characterized by variable congestion and edema of the blind spot which may be associated with periapt pillory candle flame hemorrhages. Papillitis could also be secondary to infective agent infections and possibly happens because of the reaction. Typically, it manifests itself one to a few weeks when virus infection with acute and severe Av loss that will be bilateral, however the visual prognosis is surprisingly good even in the presence of vital edema of the blind spot. Spontaneous recovery of vision might occur, requiring no treatment in most patients. In refractory cases the utilization of oral corticosteroids becomes a treatment possibility, since optic polyneuritis probably occurs due to the medicine reaction, however its use is disputed.

Optic inflammation related to dandy fever\} is incredibly rare despite the high prevalence of this disease in tropical and semitropical countries and in literature the cases according are least (10). The optic inflammation has been sometimes bilateral with dandy fever however in our case the patient given with unilateral optic inflammation. Inflammatory changes within the vascular epithelial tissue leading to vascular run, hemorrhage and anemia are often seen within the cells infected with the dandy fever virus. The cause for the optic pathology has been unknown. ?ere is proof of optic inflammation being immunogenic in origin [11] and so the treatment with steroids is even. However, the clinical general condition must be thought-about because it is to be used with caution in acute vermeil. Visual recovery, in the form of improvement of signs and symptoms, usually corresponds to rising blood platelet levels however might take many weeks to succeed in a gentle state. In according cases within the literature, the visual recovery varies from complete improvement to permanent visual loss however in our case the patient Tim proved with the steroid therapy with antibiotic cowl to $6 / 9$.

\section{Conclusion:}




\section{The American Journal of Applied}

\section{sciences}

VOLUME01 ISSUE01

Patients presenting with optic polyneuritis should be inquired and investigated for dandy fever infection and should be frequently followed up for ophthalmic examination.

\section{REFERENCES}

1. Lim WK, Mathura R, Kho A, Yoho R, Chee SP. Ocular manifestations of dandy fever. Medical specialty.

2. Angibaud G, Lunate J, labile M, Gautier C. Brain involvement in dandy fever. J Cline Neurosci.

3. Haritoglou C, Schulz F, Bialasiewicz A, Klaus V. Ocular Manifestation bee Dandy fever -Fiber. Ophthalmology.

4. Gild. Fever based on analysis of 1241cases. Arch Ophthalmic. 